\title{
"L'espace et les allusions de l'inconnu dans le premier théâtre de Maurice Maeterlinck"
}

\author{
Par \\ Noha Abdel Aziz Rizq* \\ noha.mabrouk@art.aun.edu.eg
}

\section{Résumé}

Maeterlinck a obtenu le prix Nobel de littérature comme un couronnement d'une carrière singulière pendant plus de soixante ans. Il est un symboliste célèbre. Ses théories sur le drame ont une influence décisive sur l'évolution de la transformation de la conception du théâtre. Les critiques le considèrent le nouveau Shakespeare et sa pièce est un chef-d'œuvre moderne. Il a passé son enfance à Gand, une grande ville déchue de son ancienne splendeur. Tout y respire la décadence, la vie étroite et monotone de la province. Dans cette atmosphère funèbre et triste, Maeterlinck a peur de cette vie qui lui paraît bizarre et de l'Inconnu menaçant qui influence la destinée humaine. Il tremble parce qu'il se sent, à chaque pas, guetté par un événement redoutable. Mais ces craintes reparaissent en nous sous la forme de superstitions ou des pressentiments. Il est dominé par l'angoisse des forces inconnues. L'espace est le premier élément symbolisant l'insolemment, le silence, et la peur dans son théâtre où l'Inconnu devient un être matériel. Cet Inconnu prend souvent l'image de la mort qui remplit toute l'œuvre de Maeterlinck. Cette étude est consacrée à étudier l'espace et les allusions de l'Inconnu qui envahit le premier théâtre de Maeterlinck.

* Professeur adjoint, Département de Français, Faculté des Lettres, Université d'Assiout

(L'espace et les allusions de l'inconnu...) Dr. Noha Abdel Aziz Rizq 


\section{Introduction :-}

Maurice Maeterlinck (1862 - 1949) a obtenu le prix Nobel de littérature comme un couronnement d'une carrière singulière pendant plus de soixante ans. Il est un poète symboliste célèbre, et un dramaturge génial. Ses théories sur le drame ont une influence décisive sur l'évolution de la transformation de la conception du théâtre ${ }^{1}$. Les critiques considèrent que :

\section{"L'auteur belge est un nouveau Shakespeare et que sa pièce est un chef-d'auvre moderne : l'auvre la plus géniale de ce temps. "2}

Maurice Maeterlinck a passé toute son enfance à Gand, une grande ville déchue de son ancienne splendeur. Tout y respire la décadence, la vie étroite et monotone de la province. De magnifiques monuments situés au bord des canaux et des rivières rappellent la splendeur ancienne. Autour de ces monuments se répand une atmosphère d'abandon avec une odeur presque en ruine. Par les jours pleins de brume ou de pluie, le poète se trouve, solitaire, renfermé dans la serre chaude de son âme. Ce visionnaire, derrière chaque événement aperçoit des intentions extra-humaines; derrière chaque événement anti-humains. ${ }^{3}$

Dans cette atmosphère funèbre et triste, le poète trouve la vie étrange. Il a peur de cette vie qui lui paraît bizarre, et de l'Inconnu menaçant que referme la destinée humaine. Il tremble 
parce qu'il se sent, à chaque pas, guetté par un événement redoutable. Mais ces craintes reparaissent en nous sous la forme de superstitions ou des pressentiments.

L'espace est le premier élément symbolisant l'isolement, le silence, et la peur dans le théâtre de Maeterlinck. Il est aussi une "métaphore d'un endroit où il devient possible de retrouver la mémoire de soi"t.

Avec Le Massacre des Innocents, Maeterlinck s'est montré tout pénétré du sens de l'énigme invisible. Il est dominé par l'angoisse des forces inconnues. Paul Gorceix affirme que le Gantois " n'a fait que tenter de percer l'énigme l'existence, alors que le questionnement sur l'Inconnu nous apparaît comme une sorte d'invariant de sa réflexion ${ }^{\prime 5}$

Il a donné dans cet ouvrage les deux éléments de sa conception philosophique : le mystère impénétrable des puissances de l'Inconnu et l'impuissance effrayée de l'homme devant leur bienveillance. Il a écrit lui-même dans sa préface du théâtre:

"On y a foi [dans ce drame] à d'énormes puissances invisibles et fatales, dont nul ne sait les intentions, mais que l'esprit du drame suppose malveillantes, attentives à toutes nos actions, hostiles au sourire, à la vie, à la paix, au bonheur. ${ }^{\prime 6}$

(L'espace et les allusions de l'inconnu...) Dr. Noha Abdel Aziz Rizq 
Selon l'esprit de Maeterlinck combattre les puissances formidables et hostiles dont se peuple l'Inconnu.

Durant sa vie, notre auteur sent toujours la présence redoutable de cet Inconnu autour de lui et en lui. Son âme est perpétuellement une âme angoissée et inquiète. Jules Huret nous dit :

"Il parle à voix douce et comme voilée en phrases très courtes, avec une hésitation qu'on dirait maladive, comme s'il a vraiment peur des mots qu'ils lui font mal $^{\prime \prime}$.

L'Inconnu devient pour Maeterlinck un être matériel, une sorte de bête inconsistante qui l'enlace, dans l'ombre, de ses bras, et le fait frémir sous ses attouchements incertains. C'est précisément cette espèce de sensation hallucinatoire qui constitue la nouveauté et le sortilège extraordinaire de son talent :

"La terreur de l'inconnu où nous vivons, tel a été pour M. Maeterlinck le point de départ : c'est le sentiment qui a donné l'éveil à sa faculté poétique et qu'il s'est efforcé de faire passer en nous. ${ }^{8}$

Cet Inconnu prend souvent l'image de la mort qui remplit toute l'œuvre de Maeterlinck et la domine. En effet, 'la mort au visage inconnu, est omniprésente dans l'univers Maeterlinckien 
où l'homme, comme un aveugle ne saurait atteindre la vérité qu'en acceptant de fermer ses yeux de chair pour accéder à un autre regard, celui de l'âme".9

La mort représente l'ensemble des forces occultes, qui préparent

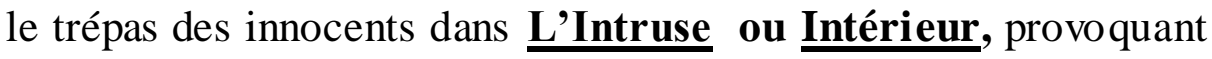
la ruine de l'amour dans Pelléas et Mélisande ou Alladine et Palomides. Selon Bruckert Thomas, le théâtre de Maeterlinck met singulièrement au jour :

"L'invu, l'invisible, l'indicible, l'inconnu, notions qui sont autant de questions et qui entraînent au théâtre celles de l'irreprésentable et de l'injouable. Ces notions ne sont pas toutes égales pour Maeterlinck, mais la lecture de son premier théâtre appelle ou engage nécessairement chacune à être pensée $" .10$

Sans doute la mort représente dans Serres Chaudes un des principaux sujets de la méditation de Maeterlinck au point de devenir chez lui obsessionnelle. L'imagination de l'auteur semble se représenter assez rarement la mort violente :

"La mort se cache derrière chaque geste, chaque mot, chaque bruit. Tout contribue à créer une atmosphère propice à déclencher sur toute l'étendue du discours théâtral un mécanisme de signification qui a la mort pour objet. " 11

(L'espace et les allusions de l'inconnu...) Dr. Noha Abdel Aziz Rizq 
Mais l'auteur présente les images de la mort qui sont celles de la déchéance. On retrouve dans ses poèmes "une odeur douceâtre de pourriture, celles des salles d'hôpitaux, lourdes de fièvres, de maladies et annonciatrices de la mort. ${ }^{12}$

Voyons par exemple ces vers expressifs dans $\underline{\text { Serres }}$ Chaudes Poèmes:

"Allez ensuite à ceux qui vont mourir.(...)

Ils sont pâles comme de malades qui écoutent pleuvoir placidement sur les jardins de l'hôpital ;"13

On peut multiplier ces images nocturnes auxquelles répondent un certain nombre de poèmes portés à leur tour par l'attente de la mort.

La même imagination de la mort envahit le premier théâtre de Maurice Maeterlinck. On assiste à la mort lente par le poison puis violente de la fragile Maleine, à l'attente anxieuse de la grande Inconnue dans l' Intruse.

Cette imagination de la mort, en effet, a évolué au cours : d'effrayante figure d'allégie médiévale constamment menaçante et implacable en une idée métaphysique et intellectualisée. Chez Maeterlinck la mort est le couronnement de l'absurdité de la vie. Aucune action humaine ne provoque son intervention, aucun 
personnage n'est inutile. Cette attitude aboutit chez Maeterlinck au «drame statique » qui ne repose plus sur l'action, mais sur la situation existentielle de l'homme dans l'univers. Autrement dit : le théâtre traditionnel ne s'intéresse pas à l'invisible, ce quelque chose d'infini que Maeterlinck considère toujours mêlé "aux actions des hommes "14 Il est convaincu que "Nous ne sommes qu'un mystère et ce que nous savons n'est pas intéressant ${ }^{\prime 15}$. Ce qu'on croit être le point de finir est peut-être le point de commencer. C'est le commencement d'une autre vie éternelle. Pour cela, l'auteur cherche toujours de présenter les signes de l'invisible qui existent partout. C'est la fatalité dont 1'homme ne peut pas fuir. L'objectif dans son premier théâtre "est de montrer l'hom me dans sa totale impuissance en face des "fatalités trop confuses » du destin dont le mécanisme reste impénétrable à l'entendement. "16

Pour Maeterlinck 'l'invisible est toujours inséré dans la réalité concrète $^{\prime 17}$ c'est pourquoi, il s'efforce "d'associer ces deux composantes en se faisant le signe de l'occulte, le vecteur du secret, afin de tirer le théâtre de son piétinement psychologique et de sa limitation au moi visible ${ }^{\prime 18}$ Il crée ce qu'on appelle le drame de situation-destin où il présente la totale impuissance et la passivité de l'homme devant la fatalité. C'est le drame statique où le dramaturge ne s'intéresse pas à présenter l'homme dans un moment extraordinaire de l'existence mais il s'intéresse à 
présenter l'existence elle-même. C'est ce que Maeterlinck adapte dans son premier théâtre :

"La princesse Maleine, sa première pièce (1980), correspond à l'application du nouveau schéma, même s'il y subsiste encore un minimum de mouvement, ne serait-ce que dans la succession des saynètes situées dans des lieux différents jardin, forêt, village, cimetière, tour ou château. "19

\section{Le décor:}

Maeterlinck, pour attirer l'attention du lecteurspectateur et pour rendre sensible la marche lente de l'inconnu, a choisi un cadre scénique qui prend une valeur caractéristique en traduisant le drame de l'invisible. Chez lui, le décor efficace et remarquable joue un rôle très important. Il est vrai que le décor semble animé et qu'il participe à l'œuvre autant que les protagonistes. Gaston Compère affirme que :

"Parler du décor chez Maeterlinck, c'est parler de tous les éléments du spectacle ${ }^{20}$

Pour la première fois peut-être dans l'histoire du théâtre français le décor, lui aussi, va participer à l'action : tout y était signe, pressentiment, présence cachée du destin.

(L'espace et les allusions de l'inconnu...) Dr. Noha Abdel Aziz Rizq 
Le décor des drames maeterlinckiens est généralement un vieux château sombre et triste ou bien une salle assez sombre en un vieux château. Les Sept Princesses se passent dans un château. Les Aveugles dans une très ancienne forêt septentrionale; Intérieur dans un vieux jardin planté de saules. $\mathrm{Au}$ fond une maison dont trois fenêtres au rez-de-chaussée sont éclairées. Selon Natacha Lafond le décor, est le champ symbolique chez Maeterlinck, il est "comme structure signifiante, (qui) se substitue la simple toile de fond. Cette dramaturgie de l'image s'appuie enfin sur une nouvelle attention accordée au silence et paysages sombres d'un Spilliaert qui interroge la place de ces êtres dans l'univers et dans les profondeurs oniriques du tragique quotidien. ${ }^{\prime 21}$

En effet, ce décor est emprunté des souvenirs que l'auteur avait gardés de son enfance, du milieu où il a été vécu. Cet énorme château, situé au bord de la mer, est bâti sur de vastes grottes mystérieuses où des eaux souterraines coulent en travaillant sournoisement à le détruire. Il y a aussi des parcs sombres avec des fontaines, des tours en ruine. L'air des marais encadre le vieux château et les émanations des grottes empoisonnent l'atmosphère et la rendent irrespirable. Ces mauvaises odeurs contribuent efficacement à donner une impression de malaise, de maladie et de mort. 
Tous ces endroits ne sont le plus souvent éclairés que par un crépuscule timide. En fait, le soleil n'est point ami du mystère et l'inconnu ne marche que dans la nuit. Dans l'intérieur des appartements, des lampes indécises luttant faiblement contre les ténèbres et parfois s'éteindront avant la fin du drame.

En effet, on peut dire que le décor chez Maeterlinck n'est pas un objet ou une facture de description matérielle :

"(Le décor est) au même titre que la musique, comme une source d'évocation et de suggestion spirituelle. Il ne matérialise pas un lieu. Il traduit une atmosphère. Il rend l'âme sensible aux mystérieux rapports des êtres et des choses. ${ }^{22}$

\section{$\underline{\text { Le château : }}$}

Parmi les principaux éléments du décor maeterlinckien vient le château. Celui-ci est l'image de la tombe d'où "l'on dirait qu'il est habité par des mots". ${ }^{23}$ On le retrouve dans tous les drames de son premier théâtre. Golaud le décrit ainsi :

$$
\begin{aligned}
& \text { "Il est vrai que ce château est très vieux et très } \\
& \text { sombre.....il est très froid et très profond." }{ }^{24}
\end{aligned}
$$

Une autre image presque identique se trouve dans les Aveugles. La vieille Aveugle :

(L'espace et les allusions de l'inconnu...) Dr. Noha Abdel Aziz Rizq 
"On dirait que c'est un château vieux très sombre et très misérable, on n'y voit jamais de lumière si ce n'est dans la tour où se trouve la chambre du prêtre. ${ }^{\prime 25}$

Ce château est particulièrement décrit dans Alladine et Palomides:

"Je ne puis m'empêcher d'être inquiète quand je rentre au palais, dit Alladine .... Il est si grand et je suis si petite, et je m'y prends encore....Et puis toutes ces fenêtres sur la mer ....on ne peut les compter .....Et les corridors qui tournent sans raison; et d'autres qui ne tournent pas et qui se perdent entre les murs....Et les sables où je n'ose pas entrer ........ ",26

Elle continue :

"On dirait que je n'ai pas été faite pour l'habiter ou qu'il n'a pas été bâti pour moi.... Une fois, je m'y suis égarée... J'ai poussé trente portes avant de retrouver la lumière du jour...et je ne pouvais pas sortir; la derrière porte s'ouvrait sur un étang...et les voûtes qui ont froid tout l'été ; et les galeries qui se replient sans cesse sur elles-mêmes 
...il y a des escaliers qui ne mènent nulle part et des terrasses d'où l'on ne perçoit rien ..."27

L'atmosphère du château pèse sur l'âme des personnages. Les héroïnes surtout se sentent mal à l'aise - et sous sa lourde oppression, elles ressentent une inquiétude, une angoisse qui n'est pas purement physique. Rappelons le passage où Mélisande confesse à Golaud qu'elle n'est pas heureuse dans le château :

"Je vais mourir si l'on me laisse ici ..."

Et encore Golaud :"Mais dis-moi quelque chose ; n'importe quoi je ferai tout ce que tu voudras ...

Mébsand : oui, oui ; c'est vrai ....On ne voit jamais le ciel clair ...Je l'ai vu pour la première fois ce matin......

Golaud : c'est donc cela qui te fait pleurer, ma pauvre Mélisande ? - ce n'est donc que cela ? ${ }^{29}$

Malgré sa masse énorme, le château n'est pas solide. Il semble même s'écrouler, mais personne n'y fait attention. C'est ainsi que Ygraine, sœur de Tintagiles, le décrit :

"Il est au plus profond d'un cirque de ténèbres...Il tombe en ruines et personne n'y prend garde ....les murailles se fondent et l'on dirait qu'il se dissout dans les ténèbres... "130 
En fait, on ne sait rien sur le pays où se trouve ce château, on sait seulement que c'est une région de brouillards. Quand le ciel n'est pas sombre et menaçant, la brume le cache et produit un effet général de flou, de lumière diffusée, d’imprécision. C'est une note constante chez Maeterlinck qui donne à son œuvre cet aspect voilé tout à fait caractéristique nous avons pu remarquer à propos d'Intérieur. Les sept princesses cherchent un peu le soleil, mais il n'y en a presque pas :

"Il y en avait un peu sur le canal ce matin, mais les arbres sont trop grands; il y a trop d'ombres; il n'y a que l'ombre ....il y a trop de brouillards et le ciel n'est jamais clair. ${ }^{131}$

Mélisande sent son cœur se serrer quand elle aperçoit de la terrasse du château où elle vient d'arriver, l'horizon gris et la mer d'où la brume monte lentement comme pour les isoler davantage.

D'où vient l'idée de ce château ?

Paul Gorceix rappelle que "Maeterlinck a fréquenté la terrasse d'Elsenôr et le château d'Inverness. ${ }^{\text {"32 }} \mathrm{Si}$ le château de La Princesse Maleine, enveloppé dans les terribles remous de la tempête, les pièces postérieures Pellés et Mélisande, Alladine et

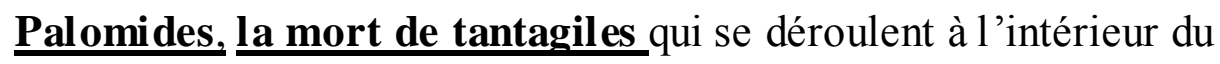
palais ne manquent pas d'évoquer l'atmosphère inquiétante et caractéristique d'Edgar Allan Poe. De celui-ci Maeterlinck s'est 
"inspiré en ce qui concerne l'atmosphère sinistre de certains de ses drames, mais cela dans une moindre mesure $" .{ }^{33}$

Par contre, il est également des palais féeriques à l'horizon bleuté et aux salles merveilleuses. En effet, le décor de la cité d'Arte velde avec son château des comtes dont la masse lézardée plonge dans l'eau noire, a bien impressionnée le poète et lui inspire l'atmosphère lugubre de ses premiers drames.

Pour Maeterlinck, le palais fournit non se ulement un cadre de rêve en facilitant le passage du quotidien dans l'irréel, il est avant tout symbole: d'accessoire du rêve. Il devient un rêve autonome, libéré, expression de l'irrationnel, du mystère de l'âme. Le palais, dans ses drames, n'est pas un simple motif littéraire, il correspond à un tropisme profond de son être. ${ }^{34}$

Les personnages qui habitent ce château vivent toujours sous la menace constante d'un écroulement : les fondations sont rongées par des eaux souterraines. Golaud dit à Pelléas :

"Avez-vous remarqué ces lézardes dans les murs et les piliers des vô̂tes? -Il y a ici un travail caché qu'on ne soupçonne pas, et tout le château s'engloutira une de ces nuits, si l'on n'y prend pas garde. ${ }^{135}$

Le château de Tantagiles et ses sœurs est décrit par Ygraine :

(L'espace et les allusions de l'inconnu...) Dr. Noha Abdel Aziz Rizq 
"Il est noir en effet ... Il est plus profond d'un cirque de ténèbres ...mais ils ont préféré le mettre de la vallée, et l'air ne descend pas en bas ... Il tombe en ruines et personne n'y prend garde... les murailles se fondent et l'on dirait qu'il se dit soût dans les ténèbres ... Il n'y a qu'une tour que le temps n'attaque point ... Elle est énorme, et la maison ne sort pas de son ombre ... ${ }^{36}$

Ici, l'habitat a une valeur allégorique : le châte au en ruines est la demeure des hommes, la terre indestructible qui le couvre de son ombre est la demeure des forces invisible, de la mort.

\section{La Tour:}

Maeterlinck confère aussi à la tour un sens symbolique : cette solitude dangereuse où l'âme se retire pour go ûter sa douleur. En réalité les tours ne sont pas absentes dans les drames de Maeterlinck; elles se présentent comme éléments d'un décor suggéré par les paroles des personnages. Ainsi dans La Princesse Maleine à la scène quatre de l'acte premier, on voit Maleine et sa nourrice prisonnières dans cette tour qui les a sauvées de la mort. Pour se délivrer, la nourrice essaye de desceller les pierres de cette tour pour que Maleine puisse s'échapper et rejoindre le prince Hjalmar. La Nourrice se lamente :

"Voilà trois jours que je travaille à desceller les pierres de cette tour, et je n'ai plus d'ongles au bout de mes pauvres

(L'espace et les allusions de l'inconnu...) Dr. Noha Abdel Aziz Rizq 
doigts. Vous pourrez vous vanter de m'avoir fait mourir. Mais voilà, il fallait désobéir ! Il fallait vous échapper du palais : il fallait rejoindre Hjalmar ! Et nous voici dans cette tour; nous voici entre ciel et terre, au-dessus des arbres de forêt! Ne vous avais- je pas avertie, ne vous l'avais-je pas dit? Je connaissais bien votre père !-Mais est-ce après la guerre qu'on nous délivrera ?". ${ }^{37}$

La princesse Maleine et sa nourrice ont passé quelques jours dans cette tour qui est véritablement un tombeau. Aussi à la scène cinq $\mathrm{du}$ troisième acte, c'est l'aspiration absolue au repos, le roi souhaite laisser ce monde matériel, plein de péchés :

"Je voudrais m'en aller où ! N'importe où ! N'importe où ! je voudrais voir les tours s'écrouler dans l'étang!"138

Dans Pelléas et Mélisande l'image de la tour prend un sens suggestif : c'est l'endroit où Golaud reçoit la réponse de son père le roi Arkël qui lui indique le chemin au château d'Allemonde. Dans sa lettre à Pelléas, s'explique :

"S'il (Arkël) consent néanmoins à l'accueillir comme il accueillerait sa propre fille, le troisième soir qui suivra cette lettre, allume une lampe au sommet de la tour qui regarde la mer. Je l'apercevrai du pont de notre navire; sinon j'irai plus loin et ne reviendrai plus .... ${ }^{39}$ 
La même image se retrouve au quatrième acte: Pelléas voudrait que personne ne le voit avec Mélisande, c'est pourquoi il a demandé de rester dans l'ombre derrière le tilleul :

"Pelléas : viens ici; ne reste pas au bord du clair de lune ...viens ici. Nous a vons tant de choses à nous dire...viens ici dans l'ombre du tilleul.

Mélisande : laissez-moi dans la clarté...

Pelléas : on pourrait nous voir des fenêtres de la tour.

Viens ici; ici, nous n'avons rien à craindre

Prends garde; on pourrait nous voir ...

Mélisande : je veux qu'on me voie... ${ }^{40}$

Dans la Mort de Tintagiles, la tour est énorme, elle cache derrière elle toute la maison. Elle n'est jamais attaquée parce que la Reine y habite :

"Ygraine: Il n'y a qu'une tour que le temps n'attaque point ... Elle est énorme, et la maison ne sort plus de son ombre...

Tintagiles: Il y a quelque chose qui s'éclaire, sour ygraine ...vois-tu, vois-tu, les grandes fenêtres rouges?

Ygraine : ce sont celles de la tour, Tintagiles, ce sont les seules où tu verras de la lumière, et c'est là que se trouve le trône de la reine ${ }^{\prime \prime 41}$ 


\section{La Forêt : -}

Malgré que l'espace est toujours clos et indéfini dans le premier théâtre de Maeterlinck, mais aussi lorsque l'action se passe dans des lieux ouverts, ces lieux sont clos. Ils sont un symbole d'une prison menaçante, de l'isolement, de l'éloignement et de la clôture. ${ }^{42}$

Influencé par le grand dramaturge anglais, Williams Shakespeare, Maeterlinck multiplie les tableaux; au cours de chaque acte, il utilise plusieurs décors qui nous transportent, selon son gré et selon les besoins de l'intrigue d'un château énorme à une forêt très sombre. Cette forêt est toujours vieille, obscure et étrange. La princesse Maleine s'écrie "Oh! Qu'il fait noir ici!" ${ }^{43}$

Le bois étrange effraye Hjalmar :

"Je n'ai jamais vu ce bois d'automne plus étrange que ce soir. (...) Je n'ai jamais vu plus de présages que ce soir Elle est là !' ${ }^{44}$

L'image de la forêt dans les Aveugles est extrêmement expressive :

"Une très ancienne forêt septentrionale, d'aspect éternel sous un ciel profondément étoilé. (....) De grands arbres funéraires, des ifs, des saules pleureurs, des cyprès, le couvrent de leurs ombres fidèles. Une touffe de longs

(L'espace et les allusions de l'inconnu...) Dr. Noha Abdel Aziz Rizq 
asphodèles maladifs fleurit non loin du prêtre dans la nuit. Il fait extraordinairement sombre, le clair de lune qui, çà et là, s'efforce d'écarter un moment les ténèbres des feuillages. ${ }^{\prime 45}$

Dans Le cahier bleu, on retrouve cette annotation si singulière :"Les Français dans le salon, les Allemands dans la forêt, les Anglais dans le jardin, les Flamands dans la maison. ${ }^{46}$ Cette réflexion éclaire bien le rapport subjectif que Maeterlinck établit entre la forêt et le peuple allemand. Il y a aussi une relation intime entre l'idée de la forêt germanique et la forêt qui revient souvent dans ses drames. C'est en effet que la forêt contribue à prêter à la pièce sa densité, sa couleur et son climat onirique. Cette forêt qui n'est jamais décrite, mais évoquée en quelques traits qui souvent font partie des indications scéniques, est pourtant présente ou sous-jacente. ${ }^{47}$

Dans les drames de Maeterlinck, la forêt n'est pas seulement un simple décor mais elle a une véritable valeur; elle est la source de l'instinct et le garant de la véritable originalité. La forêt est le lieu où se cache le mystère de la nature. Les voix plaintives des aveugles se plongent et trouvent un écho dans le chœur frissonnant de la forêt les vols d'oiseaux nocturnes entre les ramages, les rumeurs de feuilles mortes, et les bruits de la neige qui tombe.

(L'espace et les allusions de l'inconnu...) Dr. Noha Abdel Aziz Rizq 
Dans la forêt Maleine et sa nourrice ont rencontré les trois pauvres qui leur ont annoncé la mort des parents de Maleine. A la vue de ces pauvres, Maleine est tombée en peur et en crainte majeure. La forêt porte toujours en elle la mort de ses parents. ${ }^{48}$ C'est la même idée de la tristesse et de la mort qu'on retrouve dans Pelléas et Mélisande. "Et quelles forêts, quelles forêts autour du palais!" ${ }^{49}$ s'exclame Mélisande dès son arrivée au château d'Allemande. Et Golaud avoue, devant la détresse inexorable de sa femme :

\section{"Et la campagne semble bien triste aussi avec toutes ces vieilles forêts sans lumière..." ${ }^{50}$}

En général, la forêt hante l'imagination de Maeterlinck. Celui-ci a choisi son atmosphère pour placer des scènes entières : ainsi la scène deux de l'acte premier, dans Pelléas et Mélisande, a eu lieu dans une forêt. Celle-ci est le labyrinthe jardin d'amour ; elle est aussi la recherche du moi et le lieu privilégié de la rencontre avec quelques choses dangereusement différentes, Golaud dit :

"Je ne pourrai plus sortir de cette forêt. Dieu sait jusqu'où cette bête m'a mené. Je croyais cependant l'avoir blessée à mort; et voici des traces de sang. Mais maintenant, je l'ai perdue de vue; je crois que je me suis 
perdu moi-même et mes chiens ne me retrouvent plus je vais revenir sur mes pas........." 51

C'est le lieu de 1'angoisse et de la peur de l'auteur qui rappelle à la mémoire une autre forêt 'la forêt enchantée, celle de la Jérusalem libéré ${ }^{\prime 52}$ c'est vraiment l'image du Sphinx qui se présente au cavalier Golaud avec ses énigmes et ses illusions sous les trais délicats d'une petite fille qui pleure à la fontaine. Aussi dans d'autres drames de Maeterlinck le cœur de la forêt est un lieu magique, privilégie où l'homme retrouve les symboles d'une réalité qui dépasse la nature.

Quels sont les traits caractéristiques de la forêt maeterlinckienne? La forêt, chez Maeterlinck, est rarement bienfaisante, mais presque toujours à associée à l'obscurité redoutable inquiétante pour Maline et sa nourrice. Elles tombent en proie de l'angoisse ; les loups et les sangliers y rôdent, quand cette forêt n'est pas la proie du déchainement de l'étroite correspondance avec le destin des personnages :

"Maleine : connais-tu le chemin, nourrice?

La nourrice : le chemin? Ma foi, non; Je ne connais pas le chemin. Croyez-vous que je connaisse tous les chemins? Vous avez voulu aller à Ysselmonde; moi, je vous ai suivie; et voilà où nous en sommes depuis douze heures que vous me promenez dans cette forêt, où nous 
allons mourir de faim, à moins que nous ne soyons dévorées par les vers et les sangliers; et tout cela pour aller à Ysslmonde où vous serez bien reçue par le prince Hjalmar quand il vous verra venir; la peau sur les os, pâle comme une fille de cire et pauvre comme une qui n'a rien du tout $^{\prime \prime 53}$

La forêt isole le reste du monde et constitue un véritable lieu magique et propice au merveilleux de l'amour ou à l'effroi de la mort. Elle engendre le sentiment de solitude dans Pelléas et

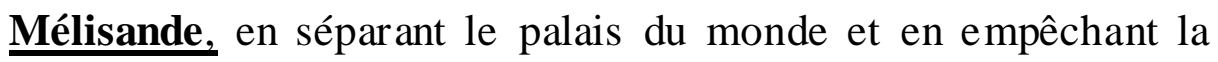
lumière de pénétrer dans les jardins.

Dans Alladine et Palomides, 'la forêt, dit Palomides, est plus belle, plus ancienne et plus grande que toutes celles que vous avez vues. Elle est pleine d'arbres et de fleurs qui sont nées d'elles-mêmes. ${ }^{154}$

De même dans Pelléas et Mélisande, la destinée de ses frondaisons et d'obscurité qui y règne délimitent la zone de l'étrange. Lieu d'épouvantes, la forêt est aussi celui du surnaturel, du fantastique. Elle ramène l'individu au plus profond de luimême et le place face à son destin. Les personnages, qui se meuvent dans sa sphère sont isolés par elle de la vie quotidienne. Ils sont solitaires et prisonniers de leur destin. 
La forêt aide aussi à constituer le vase dans lequel s'accomplit le drame. L'atmosphère, insolite et hostile des Aveugles, est caractéristique en ce sens. La forêt maeterlinckienne est celle des angoisses et des émerveillements qui s'emparent de ceux qui s'y égarent. Elle incarne un aspect de l'inconscient, dissimulant l'aventure de l'existence et de l'âme par un abîme empli des puissances obscures murmurant de voix. La forêt étrange, primitive et originelle dans $\underline{\text { Aveugles }}$ est en quelque sorte la transition avec le surnaturel, la matérialisation du fantastique et des troubles poussés de l'inconscient. C'est son climat qui contribue à rendre crédible 1 'action insolite du drame :

l'assassinant de Maleine, l'aventure de Pelléas et Mélisande, Uni vers touffu, obscur et toujours tragique, la forêt chez Maurice Maeterlinck se prête admirablement à symboliser les profondeurs de l'invisible et leur inépuisable richesse. La forêt de Maeterlinck exerce un pouvoir suggestif et magique.

\section{La forêt, Océan, et la mer :-}

Océan et forêt sont les deux principaux éléments dont le château est entouré, dans les drames du premier théâtre, à l'exception de L'Intruse et Intérieur. Chacun de ces deux éléments joue un rôle dont le but, semble opposé à celui de l'autre. Chacun tend à posséder une vertu symbolique. A l'instar de la demeure toute entière, le rôle des forêts est de créer une atmosphère oppressante et étrange, par leur profondeur, leur vieillesse, leur 
obscurité. On les croit complices du destin malheureux des personnages. Elles cernent le château et semblent l'isoler du reste du monde. Au contraire, la mer ouvre, presque toujours, l'espace aux personnages et amène dans le château la clarté qui lui semble refusée. Dans Pelléas et Mélisande, on lit cette phrase expressive :

"Geneviève : regardez de l'autre côté, vous a vez la clarté de la mer..." ${ }^{155}$

Avec quelle finesse le dramaturge s'empare de ces éléments pour dévoiler le destin de ses personnages ! De quel côté Maeterlinck va-t-il faire venir le jeune homme ? Du côté de la clarté :

"Geneviève : Pelléas, est-ce toi?

Pelléas : Oui, ...Je venais du côté de la mer....." ${ }^{56}$

Cette clarté, qui vient du côté de la mer, amène Mélisande vers son destin heureux.

La forêt, qui sépare les héros du monde et les enferme dans leur propre situation, est tout opposée à la mer. Celle-ci est l'unique lien avec le monde réel et lointain. Elle est la source de la lumière et de la clarté. L’image de la mer met un accent d'optimisme dans la nature. La jeune Aveugle :

"C'est trop loin d'ici; c'est au - delà des mers. Je viens d'un grand pays...(...) Mais j'ai vu le soleil et de l'eau et

(L'espace et les allusions de l'inconnu...) Dr. Noha Abdel Aziz Rizq 
le feu, des montagnes, des visages et d'étranges fleurs.... Il n'y en a pas de pareilles dans cette Ile; il y fait trop sombre et trop froid.......Je jouais encore au bord de la mer....." ${ }^{57}$

La forêt et la mer sont liées au problème de l'ombre et de la lumière. Le soleil est souvent exclu des drames de notre aute ur belge ; ce qui est motivé par la conception générale de l'auteur. L'atmosphère de la mort exige des ténèbres. On voit des pièces qui se passent tout entières dans les ténèbres comme $\underline{\mathbf{L} \text { 'Intruse }}$

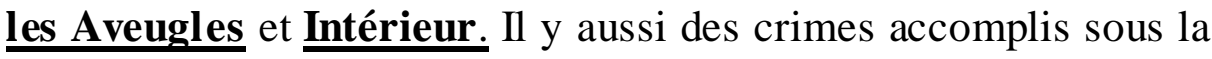
protection de l'obscurité : La lutte au château entre le roi Hjalmar et le roi Marcellus, père de Maleine (acte premier, scène première) ; la mort de la princesse Maleine de la main de la reine Anne (acte IV, scène 5); la mort d'Anne et de Hjalmar (acte V, scène 4); la mort y de Pelléas par son frère Golaud (acte IV, scène 5$)$.

\section{L’obscurité et les coul eurs :-}

L'exploration des ténèbres de l'inconnu dans laquelle s'est engagé le mystère de Rusbroeck suggère à Maeterlinck des images plus expressives. Dans la Princesse Maleine, il a utilisé le noir comme couleur clef. On voit le noir du ciel, noir de l'obscurité, noir des forêts sans lumière; le moulin est étrangement noir, le chien de Maleine est noir, les corridors du 
château sont noirs, noir des nuages. Aussi dans Pelléas et $\underline{\text { Mélisande, }}$ règne la nuit noire et froide, la mer y sombre ; lorsque Pelléas entre dans la grotte, il fait très noir; de même il fait noir sous les arbres où Pelléas et Mélisande, se sont donnés rendezvous. L'obscurité est la note dominante dans Alladine et Palomides: les fossés du palais sont remplis d'une eau noire, les arbres sont noirs et on retrouvera les deux amants enlacés au fond de l'eau noire.

En fait, le jeu des couleurs joue un rôle remarquable dans le premier théâtre de Maeterlinck. A côté du noir, il a aussi employé le bleu avec netteté. Cette couleur concourt à créer une atmosphère de rêve laissant entrevoir le mystère de l'âme humaine et de l'être. Dans les grottes d' $\underline{\text { Alladine et Palomides, }}$ le bleu domine grâce à une savante représentation de la lumière : grandes salles bleues, voûtes bleues. Cette couleur est tout éclairée par le reflet de la mer. La grotte de Pelléas et Mélisande, à son tour, est pleine de ténèbres bleus, baignée de clarté de la mer et du ciel qui se confondent.

Ces quelques exemples ont un double intérêt pour notre propos. Car, ils montrent bien que "Maeterlinck, par son sens de la couleur, se rattache à la famille des artistes flamands et qu'il est, à part entière, le représentant de la littérature flamande, germanique, extrêmement riche en images et en coloris. ${ }^{~} 58$ 


\section{$\underline{\text { Le Jardin :- }}$}

A l'entrée du château on voit le jardin, celui-ci est étroitement lié à celui-là. Maeterlinck, dans ses drames, se sert du jardin en tant que décor approprié au merveilleux. Chez lui, le jardin permet l'épanouissement des rêves en rendant crédible le fantastique. Cependant là aussi l'image existe elle-même et pour elle-même. D'abord cette image acquiert bientôt son indépendance. Le parc de Maeterlinck est généralement associé au palais; aussi dans Pelléas et Mélisande, Alladine et

\section{Palomides et Intérieur.}

Du jardin s'incline l'image du jet d'eau. Cet élément liquide appartient particulièrement au paysage dans lequel s'est déroulé l'enfance de Maeterlinck, dans la propriété d'Ostacker à Gand. Cet élément aquatique intervient dans le théâtre de Maeterlinck sous la forme de la mer, des canaux dormants, de sources ou de fontaines.

\section{La Fontaine :}

En fait, le jet d'eau apparaît dans quatre pièces de Maeterlinck: La Princesse Maleine, Pelléas et Mélisande, Alladine et Palomides et Intérieur. 
Dans La Princesse Maleine, la fontaine se trouve dans le parc où Maleine et Hjalmar se rencontrent, il est le lieu de leur rendez-vous nocturne. Hjalmar :

"Ici, près du jet d'eau. -Nous nous entre verrons à la clarté de l'eau. Il fait étrange ici ce soir. ${ }^{159}$

Au moment où Hjalmer embrasse Maleine, "le jet d'eau, agité par le vent, se penche et vient retomber sur eux. ${ }^{\prime 60}$ Lorsqu'ils se séparent, il « sanglote étrangement et meurt. »

Quant à Pelléas et Mélisande, la fontaine représente en quelque sorte un centre autour duquel se déroulent des événements décisifs. Mélisande a été découverte près d'une fontaine dans la forêt; elle perdra aussi l'anneau symbolique dans le bassin d'une fontaine. C'est aussi près d'une fontaine que les amants Pelléas et Mélisande se rencontrent de nuit et c'est sur le bord de la fontaine que Pelléas tombera mortellement frappé par Golaud.

Dans Intérieur figure aussi un jet d'eau jaissant d'une pelouse, sous le clair de lune :

"On aperçoit le ciel étoilé, le pelouse et le jet d'eau sous le clair de lune, tandis qu'au milieu de la chambre abandonnée l'enfant continue de dormir paisiblement dans le fauteuil. -silence ${ }^{\prime \prime 61}$ 
Il convient de noter dans les deux premiers exemples l'association fontaine-amour, plus exactement la naissance de l'amour, et dans le dernier celle du jet d'eau et de l'enfance. Sigmond Freud nous éclaire sur pareille rencontre. Il écrit dans son Introduction à la psychanalyse :

"La naissance se trouve régulièrement exprimée dans le rêve par l'intervention de l'eau, ce qui veut dire qu'on enfante ou qu'on naît. "'62

Dans les trois exemples cités au-dessus, eau et clarté vont de pair, et l'eau vive tranche avec le néant de l'obscurité environnante.

C'est peut-être à travers l'image du jet d'eau comme elle apparaît dans Alladine et Palomides que se révèle le mieux l'arrière-plan symbolique du thème. Le vieux Ablamore fait à Alladine cette étrange révélation :

"Qu'y a - t-il dans le parc? - tu regardais l'avenue des jets d'eau qui s'ouvre devant les fenêtres? Ils sont merveilleux et infatigables. Ils se sont élevés tour à tour, à la mort de chacune de mes filles....La nuit, je les entends chanter dans le jardin....Ils me rappellent les existences qu'ils représentent, et je puis distinguer leurs voix... "63 
Chaque jet d'eau du jardin magique représente l'âme des filles qui à travers son murmure, perçoit la voix des disparues. L'eau et l'existence, l'eau et l'âme se trouvent ainsi associées selon un symbolisme ancestral. Chez Maeterlinck, c'est l'âme des filles mortes du roi Ablamore qui vit immortellement à travers l'eau vive. Ce jet d'eau représente l'existence spirituelle ou matérielle et symbolise la présence de l'inconscient qui, sous forme liquide, jaillit des sources souterraines. C'est le symbole de la vie de l'union fondamentale de l'homme et de l'univers. Disparues charnellement, les filles d'Ablamore reparaissent devant leur père.

\section{$\underline{\text { La grotte : }}$}

Le vieux château est bâti sur des grottes souterraines. Le monde souterrain fournit au dramaturge un cadre pittoresque et propice: pittoresque du fait de sa situation inhabituelle, de l'obscurité et du silence qui y règnent en opposition au monde à ciel ouvert, propice à la technique théâtrale, car la grotte constitue un uni vers à part, clos comme le jardin, vraiment fait pour le rêve. En outre, ce royaume de la nuit et du silence où la notion du temps est absente, représente un lieu est privilégié pour les épanchements de 1 'amour $^{64} \mathrm{La}$ grande scène d'amour, entre Alladine et Palomides, se déroule sous terre elle aussi.

De plus, la grotte est un lieu favorable à l'évocation de l'étrange, du merveilleux et de l'inquiétant: elle nous donne un 
sentiment d'effroi et d'émerveillement. Pelléas montre à Mélisande l'étrangeté angoissante des grottes au bord de la mer. Dans l'Intruse et Les Aveugles la grotte n'est qu'un élément décoratif du paysage dont le rôle est de créer une atmosphère, tandis que dans Pelléas et Mélisande, Alladine et Palomides, Maeterlinck lui réserve une place centrale et lui prête la valeur de mythe.

Il est important de remarquer que, dans Pelléas et Mélisande, Alladine et Palomides, le thème de la grotte est lié étroitement à celui de l'eau. L'ambiance spéciale qui se dégage de ces lieux contribue avec beaucoup de force à rendre sensible les choses informulées qui s'agitent dans les régions subconscientes de l'être. Telle est la signification que l'atmosphère prend dans la scène où Golaud et Pelléas descendent aux souterrains du palais. De l'eau stagnante qu'il y a au fond de ces grottes monte une odeur de mort qui enveloppe tout :

"Golaud : sentez-vous l'odeur qui monte?...

Pelléas : on dirait une odeur de tombeau. ${ }^{165}$

C'est un monde inquiétant qui est évoqué, dans lequel "il ne faut pas s'engager sans guide: Il en est qui ne sont jamais revenus $^{\prime \prime 66}$, un monde où l'homme court à sa perte, Pelléas dit :

"Il y a des endroits dangereux et le sentier est très étroit, entre deux lacs dont on n'a pas encore trouvé le fond". ${ }^{67}$

(L'espace et les allusions de l'inconnu...) Dr. Noha Abdel Aziz Rizq 
C'est un univers clos et inconnu dont "on ne l'a (la grotte) pas encore explorée jusqu'au fond ${ }^{\prime \prime}{ }^{68}$ L'idée de ruine arrive à son apogée par l'engloutissement imminent du château : "Il y a ici un travail caché qu'on ne soupçonne pas; et tout le château s'engloutira une de ces nuits, si l'on n'y prend pas garde. ${ }^{\prime 69} \mathrm{On}$ relève l'association de la mort avec l'image de la nuit.

Dans Alladine et Palomides, si le décor est moins macabre, l'immersion du palais Ablamore dans la mer est cependant indiquée de façon allusive :

'Palomides: Je savais que la mer inondait les plus basses ; et c'est probablement le reflet de la mer qui nous éclaire ainsi..... "10

Il s'agit là aussi d'un monde obscure « Ils ont cru nous ensevelir dans la nuit», d'un monde labyrinthique inquiétant par succession «des grottes, salles et voûtes innommables » ${ }^{71}$ et d'un monde fermé « personne n'y descendait : et le roi seul en a les clefs $»^{72}$

En fait, la grotte symbolise l'âme humaine qui possède des régions dont il ne faut s'approcher qu'avec une très grande prudence. Les restes de passions mortes s'y trouvent aussi comme les tendresses, des sentiments éternels 
Dans Pelléas et Mélisande, après avoir perdu l'anneau qui est tombé au fond de la grotte au bord de la mer, Golaud envoie Mélisande en compagnie de Pelléas pour le chercher. Tous les deux s'enforcent dans la grotte comme dans leur destinée là, dans cette obscurité comme dans leur âme mystérieuse, dort leur amour. Ils affrontent la route périlleuse ; ils côtoient leurs âmes insondables, sans prudence, sans réflexion; ils s'en remettent à la merci des cieux. Pelléas dit à Mélisande :

"Je n'ai pas songé à emporter une torche ou une lanterne, mais je pense que la clarté du ciel nous suffira ${ }^{\prime 73}$

Cette grotte est très obscure et très grande. Elle est pleine de stalactites et de ténèbres bleues, et les hommes ne l'ont pas explorée jusqu'au fond. Elle renferme de grands trésors : ce sont toutes les richesses de la tendresse humaine. Mais ceux qui se sont engagés dans ces retraites dangereuses n'en sont pas tous revenus. On y voit des épaves d'anciens naufrages.

Qu'importe! Explorateurs tremblants et ravis, Pelléas et Mélisande avancent toujours. Pelléas hésite un instant :

"Moi-même, je n'ose pas aller trop avant. Nous nous arrêterons au moment où nous n'apercevrons plus la clarté de la mer ou du ciel. ${ }^{174}$ 
Mais cette incertitude est brève. Il sait qu'il suffit du reflet d'une petite lampe pour étoiler la voûte, où s'enchâssent des fragments de cristal et de sel, pour découvrir soudain tout un autre firmament . Il sait qu'un peu d'amour illumine la vie. Et il prend Mélisande par la main :

"Voyez-voyez, je crois que le ciel va s'ouvrir.....

Donnez - moi la main, ne tremblez pas ainsi, ne tremblez pas ainsi. Il n'y a pas de danger: nous nous arrêterons......" ${ }^{175}$

Alors, il sera trop tard. Ils se seront engagés trop loin, et brusquement le rêve chassé par une lumière blanche et crue, se dissipera. Ils se trouveront face à face avec la réalité. Car la lune spectrale entre dans la grotte d'amour, et l'on aperçoit, à une certaine profondeur, trois pauvres écoulés dans leur sommeil, contre un quartier de roc. Qui sont-ils ? On n'en sait rien, à moins que ces messagers du malheur, ces victimes de la famine, ne soient les symboles fatidiques des trois héros principaux du drame (Golaud, Pelléas, Mélisande). Car plus tard quand Pelléas égorgé, tombera dans la fontaine des Aveugles, Golaud et Mélisande blessés se traîneront jusqu'à la porte du château, et la vieille servante les trouvera, à l'aube, étendus tous les deux "tout à fait comme des pauvres qui ont faim. ${ }^{176}$ 
A côté du palais et de la grotte, Maeterlinck a découvert un décor assez poétique et qui a une signification symbolique majeure : le labyrinthe. L'insolite de l'intérieur labyrinthique a hanté son imagination.

Dans Alladine et Palomides, c'est une impression de rêve étrange et inquiétant qui naît de l'évocation d'Alladine :

"Je ne puis m'empêcher d'être inquiète quand je rentre au palais......

Et les salles où je n'ose pas entrer.... ",77

Dans La Mort de Tintagiles, Bellangère décrit aussi les corridors de la tour :

"Je n'avais jamais vu.... Il y avait d'autres corridors éclairés par des lampes ; puis des galeries basses qui n'avaient pas d'issue ....Je savais qu'il était défendu d'avancer ....J'avais peur et j'allais revenir sur mes pas, quand je surpris un bruit de voix qu'on entendait à peine.... ",78

D'autre part, Maeterlinck va donner au labyrinthe une charge symbolique. Il se présente comme un symbole adéquat pour celui que le monde de l'inconnaissable ne cesse de hanter. Palais érigés sur des galeries souterraines, à l'inquiétante profondeur, grotte obscures et labyrinthiques, sont autant 
d'images pittoresques qui se prêtent facilement à la représentation du monde intérieur et irrationnel de l'inconscient mystérieux et angoissant à la fois qui sommeille derrière la face éclairée de la conscience.

\section{$\underline{\text { Le Clair-obscur :- }}$}

Le décor maeterlinckien est très suggestif. A côté de la couleur noire, qui est très fréquente dans ces drames, il y a aussi de la lumière : le ciel bleu est comme un anneau de cristal ; l'eau est toujours source de la clarté :

\section{"Est-ce l'eau.... Elle semble plus belle et plus pure et plus bleue que tout l'océan de la terre ...." ${ }^{179}$}

Aussi l'emploi du clair-obscur donne des effets très pittoresques :

\footnotetext{
"Je ne vous vois pas. - venez ici, il fait plus claire, renversez un peu la tête vers le ciel. ${ }^{\prime 80}$
}

L'obscurité symbolise l'état d'âme des personnages de Maeterlinck ainsi que l'atmosphère d'angoisse et de crainte où ils vivent. Elle s'accorde parfaitement à l'état d'âme de l'auteur luimême. L'obscurité chez lui, devient le symbole de la mort et des puissances inconnues. 
A côté de l'obscurité, il y a des brumes, les marécages, les vapeurs d'eaux; dans La Princesse Maleine: "là-bas, dans le brouillard, du côté du cimetière ...." ${ }^{81}$. Plus loin encore : "mais qu'est-ce que toutes les femmes sur les marais $?^{82}$

Il a recours également à la clarté qui crée une atmosphère de sécurité et de sérénité. La clarté est le symbole du bonheur et de l'espérance. La lumière symbolise aussi la vérité : après avoir accompli leur crime, le roi $\mathrm{Hjalmar}$ et la reine Anne restent dans une salle d'attente à la chapelle. Tout à coup, une béguine ouvre la porte de la chapelle.

"Une grande clarté rouge provenue des vitraux et de l'illumination du tabernacle inonde subitement le roi et la reine Anne ". ${ }^{83}$

Le roi est convaincu que la lumière signifie qu'on a découvert leur crime.

La lumière, dans ces drames, perce les ténèbres et les ténèbres absorbent la lumière ainsi que le désespoir et la douleur humaine se mêlent au bonheur et au calme. De là vient le rôle du soleil qui n'est pas tout à fait exclu des drames de Maeterlinck.

Dans La Princesse Maleine, la nourrice s'écrie : "Je ne vois que le soleil!" ${ }^{84} \mathrm{Et}$ dans Pelléas et Mélisande, la servante dit: "le 
soleil se lève sur la mer" $^{\prime \prime}{ }^{85}$ Le sens symbolique de la lumière nous est le mieux révélé dans Alladine et Palomides :

"Palomides, dans l'appartement : ...Je vois un rayon de soleil qui passe par les fentes des volets.

Une des saurs : oui, il fait grand soleil au dehors. ${ }^{186}$

Ablamore, après avoir découvert l'amour d'Alladine et Palomides pense à sa vengeance. ${ }^{87}$ Dans le même acte, il fait le geste qui évoque la renonciation à son projet, il ouvre le volet et invite les deux amants à regarder le paysage :

"Voyez-vous, mes enfants ; il fait noir dans la chambre. Il y fait aussi noir qui si l'on se trouvait à mille pieds sous terre. Mais j'ouvre un des volets et voyez ! Toute la lumière du ciel et du soleil ! Il n'y faut pas un grand effort et lumière est pleine de bonne volonté .....Il suffit qu'on l'appelle; elle obéit toujours.... Avez-vous vu le fleuve avec ses petites îles entre les près en fleurs..."

On remarque que Maeterlinck utilise la lumière dans ses drames composés de plusieurs actes pour de multiples raisons : généralement pour combattre la monotonie qu'engendre presque nécessairement une suite de scènes où règne une quasi-obscurité ; plus particulièrement, pour soutenir un sentiment d'espérance. ${ }^{89}$

(L'espace et les allusions de l'inconnu...) Dr. Noha Abdel Aziz Rizq 
D'autre part Maeterlinck semble chercher à établir un subtil rapport entre l'inquiétude humaine et l'indifférence de la nature. Voyons, par exemple, dans Pelléas et Mélisande :

'Tiens, dit Pelléas, on vient d'arroser les fleurs au pied de la terrasse, et l'odeur de la verdure des roses mouillées s'élève jusqu'à nous". 90

Ces paroles évoquent un spectacle qui ne contraste pas seulement avec l'humeur sombre de Golaud, mais également avec la destinée menaçante de Pelléas que nous suggèrent les scènes précédentes.

Ce qui donne une vale ur remarquable à de pareils textes est leur étrangeté par rapport à l'esprit du drame. Si on peut trouver à cet esprit une vérité profonde, on ne peut pas nier l'étrangeté de l'atmosphère d'où il se dégage. Mais cette atmosphère on l'accepte si rapidement qu'on la tient pour normale, si bien que les courts textes cités se charge nt eux-mêmes d'étrangeté. Comme Maeterlinck enveloppe toujours l'aventure humaine d'une atmosphère conforme à celle qu'exige le dénouement de la pièce. Dans les premiers drames où la mort triomphe, la scène se trouve le plus souvent dans l'obscurité.

En effet, les décors de ces drames n'ont été créés que pour rendre susceptible la dramatisation de la vie humaine considérée dans ce qu'elle a de plus pur, de plus profond, de plus inaltérable, 
et rétrospectivement en second lieu, pour légitimer cette dramatisation. Ce décor n'est qu'un élément résultant et sa vérité réside dans la manière toute spéciale dont Maeterlinck a considéré l'être humain et l'a employé.

En un mot, le théâtre de Maeterlinck doit une partie de sa gloire à la victoire des symbolistes. La modernité de son théâtre réside dans l'atmosphère morne et l'espace décoratif qui inspire l'originalité de son théâtre, le théâtre de l'invisible qui nous guide à l'Inconnu, le destin où la mort: "il n'y a rien de rassurant, ni d'intime dans l'espace de ses pièces. Nulle précision, nulle situation ni géographique, ni historique, un château médiéval très froid et très profond, une île aux forêts impénétrables, une mer qui isole, un ciel qui prépare aux malheur"91 et les personnages sont toujours inc apables de retrouver leur chemin, ils sont prisonniers d'un espace rendu menaçant par l'Inconnu. 
- Marge :-

1. Voir Emanuela (Spinoso), « le bruit de l'indicible dans les drames de Maeterlinck et de Beckett », thèse de magistère, université de Padova, Padova, 2016. P.9.

2. Cité Andrel (Carmen), "Originalité et modernité du théâtre de Maurice Maeterlinck », Université «le Bas Danube » de Galati, Roumanie : dialnet.unirioja.es/descarge/articulo/2555093.PDF, 2007.

3. Doneux (Guy), «Maurice Maeterlinck, une poésie, une sagesse, un homme », Bruxelles, éd. Palais des Académies, 1961, P. 14.

4. Emanuela (Spinoso), « le bruit de l'indicible dans les drames de Maeterlinck et de Beckett », Op.cit., P.28.

5. Gorceix (Paul), «Maurice Maeterlinck et l'analogie», communication présentée à la séance mensuelle de l'Académie royale de langue et de littérature française de Belgique, le 12 février 2000, P.3.

6. Maeterlinck (Maurice), «Préface du théâtre 1 », Paris, Fasquelle, 1902, P.1x.

7. Huret (Jules), «Enquête sur l'évolution littéraire», Paris, Charpentier, 1891, P.118.

8. Doumic (René), «Les deux manières de M. Maeterlinck », Revue des Deux Mondes, $5^{\mathrm{e}}$ période, tome 10, http://fr.wikisource.org, consulté le 20/10/2015.

9. Jung Kwan (Hyun), «Maeterlinck et le théâtre pour marionnettes : Alladine et Palomides, Intérieur, la Mort de Tintagile » in Nineteenth-century French studies, vol.40,No.1 winter 2011-2012, P.24.

10. Bruckert (Thomas), «le théâtre de Maeterlinck: une manière du dire », Paris, Acta Fabula, vol.18 N.17,éd. Rééditions, traduction, septembre 2017, URL : http ://www.fabula.org/revue/document 10423.php. Page consultée le 20 janvier 2020.

11. Roger (vandenbrande) « la mort voilée : mise en scène et mise en langage chez Maeterlinck, ou analyse d'une registre sémantico- 
pragmatique », in «Annales tome 27 », Gand, Fondation Maurice Maeterlinck, A.S.B.L., 1989, p.37.

12. Gorceix (Paul), "Les affinités allemandes dans l'œuvre de M: Maeterlinck », Poitiers, Publication de l'université de Poitiers (lettres et sciences humaines), $1975 ;$ P.211.

13. Maeterlinck (Maurice), «Serres Chaudes-poèmes », Bruxelles, Lacomblez, 1890, P.145.

14. Maeterlinck (Maurice), "Préface au théâtre de 1901 ", Paris, Fasquelle, 3 volumes. Le «Préface » a été rééditée par Paul Gorciex dans «Maeterlinck, Serres Chaudes, Quinze chansons, la Princesse Maline », Paris, Gallimard, 1983, [2è édit, 1995] « Poésie », P.300.

15. Mac (M), « le trésor des humbles 1896, le réveil de l'âme ", dans Gorceix (Paul) « la Belgique fin de siècle. Romans-Nouvellesthéâtre (Ekhoud, Lemonnie, Maeterlinck, Rodenbach, Van lerberghe, Verhaeren », Bruxelles, éd. Complexe, 1997, P.851.

16. Gorceix (Paul), «Le défi de Maurice Maeterlinck: un théâtre métaphysique ", dans Lazzarini - (Dossin), "Théâtre tragique et modernité en Europe XIX, XXe siècles», Bruxelles, col. Documents pour l'histoire des francophonies, 2004, P. 161.

17. Ibidem.

18. Ibidem.

19. Ibid., P. 162.

20.Compère (Gaston), «Maurice Maeterlinck, prix Nobel 1911 », Bruxelles, le Cri, 2015. P. 150.

21. Voir Lafond (Natcha), «Pour une dramaturgie de l'image chez Maeterlinck », Acta Fabula, vol.9, n.9 Notes de lecture, Octobre 2008, URL : http://www.fabula.org/revue/document 4596. php, page consultée le 24 janvier 2020.

22. Bablet (D.), «Esthétique générale du décor de théâtre de 1870 à $1914 »$, cité Emanuela (Spinoso), op.cit, P.57.

23. Materlinck (Maurice), «Pelléas et Mélisand » in «Théâtre II », acte III, sc.3, Bruxelles, lacomblez, 1902, P.57

24. Ibid., acte II, sc.2, pp. $33-34$.

(L'espace et les allusions de l'inconnu...) Dr. Noha Abdel Aziz Rizq 
25. Maeterlinck (M.), « Les Aveugles », in «thêâtre I », p.312.

26. Maeterlinck (M.), « Alladine et Palomides » in « Théâtre II », acte I, P. 122.

27. Ibid., acte X, P. 123.

28. Maeterlinck (M.), « $\underline{\text { Pelléas et Mélisande } ~}$ in « Théâtre II », acte II, Sc.2, P. 32.

29. Ibid., P.34.

30. Maeterlinck (Maurice.), « La Mort de Tintagiles » in « Théâtre II », acte I, P. 207.

31. Maeterlinck (Maurice.), « Les Sept Princesses », Bruxelles, Lacomblez., 1891, P.16.

32. Groceix (Paul), "Les Affinités allemandes dans l'œuvre de M. Maeterlinck », P.261

33. Carré (J.M), «L'évolution du théâtre de Maeterlinck », Paris, Revue des Cours et Conférences, 1925-1926, p.467.

34. Groceix (Paul), "Les Affinités allemandes dans l'œuvre de M. Maeterlinck », P.261.

35. Maeterlinck (Maurice), «Pelléas et Mélisande », «théâtre II », acte III, Sc.3, P.56.

36. Maeterlinck (Maurice), « la Mort de Tintagiles », acte I, P.207.

37. Maeterlinck (Maurice), « La Princesse Maleine », acte I, P.207.

38. Ibid., P.37-99.

39. Maeterlinck (Maurice), "Pelléas et Mélisande », acte I, Sc.3, P.15.

40. Ibid.p, acte IV, Sc.4, PP.82-83.

41. Maeterlinck (Maurice), «La Mort de Tintagiles », acte I, PP.207208.

42. Voir Emanuela (Spinoso), op.cit., pp.129-131.

43. Maeterlinck (Maurice), "La Princesse Maleine », acte II, SC.I P.35. 44. Ibid., PP. 63 - 64.

45. Maeterlinck (Maurice.), «Les Aveugles », «Théâtre I », PP. 281282.

(L'espace et les allusions de l'inconnu...) Dr. Noha Abdel Aziz Rizq 
46. Maeterlinck (Maurice.), « Le cahier bleu », Bruxelles, club du livre 1977, P. 58.

47. Groceix (Paul), "Les Affinités allemandes dans l'œuvre de M. Maeterlinck », P.255.

48. Maeterlinck (Maurice), "La Princesse Maleine », acte II, Sc.1 P.36.

49. Maeterlinck (Maurice), "Pelléas et Mélisande », acte I, Sc.4, PP.18.

50. Ibid., acte II, Sc.2, P.34.

51.Ibid., acte I, Sc.2, P.8.

52. Groceix (Paul), "Les Affinités allemandes dans l'œuvre de M. Maeterlinck », P.257.

53. Maeterlinck (Maurice), «La Princesse Maleine », acte II, Sc.1 P.36.

54. Maeterlinck (Maurice), «Alladine et Palomides », acte II, Sc.2 P.128.

55. Maeterlinck (Maurice), «Pelléas et Mélisande », acte I, Sc. 4, P.18.

56. Ibid., P. 19.

57. Maeterlinck (Maurice), «Les Aveugles », P. 308.

58. Gorceix (Paul), "Les Affinités allemandes dans l'œuvre de M. Maeterlinck », P.321.

59. Maeterlinck (Maurice), « La Princesse Maleine », acte II, Sc. 6, P. 64.

60. Ibid., P.65.

61. Maeterlinck (Maurice), « Intérieur », P. 199.

62. http://fr.m.wikisource.org «page: Freud. Introduction à la psychanalyse ; 1923 » (consulté le 23/7/2018)

63. Maeterlinck (Maurice), «Alladine et Palomides », acte II, Sc.1 P.125.

64.Gorceix (Paul), «Les Affinités allemandes dans l'œuvre de M. Maeterlinck », P.329.

65. Maeterlinck (Maurice), «Pelléas et Mélisande », acte III, Sc. 3, P.56.

66. Ibidem.

67. Ibid., acte II, sc.3, P.38.

(L'espace et les allusions de l'inconnu...) Dr. Noha Abdel Aziz Rizq 
68. Ibidem.

69. Ibidem.

70. Maeterlinck (Maurice), « Alladine et Palomides », acte IV, PP.154155.

71. Ibidem.

72. Ibid., P.154.

73. Maeterlinck (Maurice), « Pelléas et Mélisande », acte II, Sc. 3, P.38. 74. Ibidem.

75. Ibid., PP.38-39

76. Ibid., acte V, Sc.1, P.96.

77. Maeterlinck (Maurice), " Alladine et Palomides », acte I, P.122.

78. Maeterlinck (Maurice), "La mort de tintagiles », acte II, P.122.

79. Maeterlinck (Maurice), « Alladine et Palomides », acte IV, Sc.1, P.157.

80. ${ }^{1}$ ) Maeterlinck (Maurice), «La Princesse Maleine », acte II, Sc. 6, P.46.

81. Ibid., acte III, sc.3, P.93.

82. Ibid., P.95.

83. Ibid., acte V, sc.2, P. 196.

84. Ibid., acte I, Sc. 4, P.28.

85. Maeterlinck (Maurice), « Pelléas et Mélisande », PP.38-39

86. Maeterlinck (Maurice), "Alladine et Palomides », acte III, SC.3 P.145.

87. Ibid., P.140.

88. Ibid., P.146.

89. Ibid., acte II, S.c.1P.57.

90. Maeterlinck (Maurice), «Pelléas et Mélisande », acte III, S.c.4, P.58.

91.Emanuela ( Spinoso), op.cit. ; P.130.

(L'espace et les allusions de l'inconnu...) Dr. Noha Abdel Aziz Rizq 


\section{Bibliographie}

\section{Corpus :-}

- Maeterlinck (Maurice),

- « Théâtre I, la Princesse Maleine 1890, l’Intruse

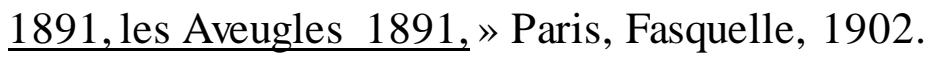

- « Théâtre II, Pelléas et Mélisand 1892, Alladine et $\underline{\text { Palomides }}$ 1894, La Mort de Tintagiles 1894, Intérieur 1894 » Bruxelles,

Lacomblz, 1902.

- « $\underline{\text { Serres Chaudes-Poèmes }}$, Bruxelles, Lacomblez , 1890.

- «Les Sept Princesses », Bruxelles, Lacomblez, 1891.

- « le Cahier Bleu $»$, Bruxelles, club du livre Mois, 1977.

\section{Euvrages généraux :-}

- Huret (Jules), «Enquête sur l'évolution littéraire », Paris, Charpentier, 1981.

(L'espace et les allusions de l'inconnu...) Dr. Noha Abdel Aziz Rizq 
- Lazzarini (Dossin), «Théâtre tragique et modernité en Europe XIX, XXe siècles"», Bruxelles, col. Documents pour l'histoire des francophonies, 2004.

\section{III-Cuvrages consacrés à Maeterlinck :-}

- Compère (Gaston), « Maurice Maeterlinck, prix Nobel

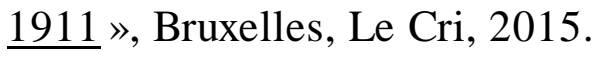

- Doneux (Guy), « Maurice Maeterlinck. une poésie, une sagesse, un homme », Bruxelles, Palais des Académies, 1961.

- Gorceix (Paul), «les Affinités allemandes dans l'œuvre de

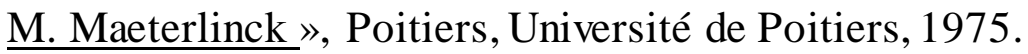

« $\underline{\text { la Belgique fin de siècle Romans- Nouvelles - théâtre }}$ Ekhoud Lemonnie, Maeterlinck, Rodonbac, Van Lerberghe, Verhaeren » Bruxelles, complexe, 1997.

\section{$\underline{\text { IV- Articles :- }}$}

- Bruckert (Thomas), «Le Théâtre de Maeterlinck: une manière du dire», Paris, Acta Fabula, vol 18, $\mathrm{n}^{\circ}$. 17, réédition, traduction, septembre 2017.

- Carré (J.M), « L'évolution du théâtre de Maeterlinck », Paris, Revue des cours et conférences, 1925-1926.

(L'espace et les allusions de l'inconnu...) Dr. Noha Abdel Aziz Rizq 
- Doumic (René), «les deux manières de M. Maeterlinck », Revue des Deux mondes, $5^{\mathrm{e}}$ Périade, tome 10

- Gorceix (Paul), « Maurice Maeterlinck et l'analogie », communication présentée à la séance mensuelle de l'Académie royale de langue et de la littérature française de Belgique, le 12 février 2000.

- Jung Kwan (Hgun), « Maeterlinck et le théâtre pour marionnettes : Alladine et Palomides, Intérieur, la mort de Tintagile », in Nineteenth- centuary french studies, vol.40, No.1 Winter 2011-201.

- Lafond (Natacha), « Pour une dramaturgie de l'image chez Maeterlinck», Acta Fabula, vol.9, No.9, Notes de Lecture, Octobre 2008.

- Roger (Vandenbrande), « $\underline{\text { La mort voilée : mise en scène }}$ et mise en language chez Maeterlinck, ou analyse d'une registre sémantico-pragmatique » in Annales, Tome 27, Gand, Fondation Maurice Maeterlinck, A.S.B.L. , 1989.

\section{V-Sciographie :}

- http://www.dialnet.unirioja.es/descarge/articulo/2555093. PDF, 2007.

- http://fr.wikisource.org, consul té le 20/10/2015. 
- http://fr.m. wikisource.org, « page : Freud-Introduction à la psychanalyse, 1923 », consul té le 23/7/2018.

- http://www.fabula.org/revue/document 4596.php,page consultée le 24/1/2020.

\section{VI- Thèses :}

- Emanuela (Spinoso), « le bruit de l'indicible dans les drames de Maeterlinck et de Beckett », thèse du magistère université de Padova, Padova, 2016. 


\section{الملخص}

حصل ماتيرلينك علي جائزة نوبل للآداب تتويجاً لمسبيرته الأبية الفريدة لأكثز من ستين عاماً. فهو شاعر رزي شهيز وكاتب مسرحي لامع ورائي متميز. وله نظريات حديثة في الدراما، تركت أثز كبير وواضح علي تطور التحول في مفهوم المسرح الحديث. ويعتبره النقاد شكسبير الجديد. وتعد مسرحياته من الأعمال الرائدة في العصر الحديث. قضي ماتيرلينك طفولته في مدينة غينت العظيمة التي تشتهر بآثارها القديمة والتي فقت رونقها وكل شئ فيها يوحي بالتهدم والقدم وفقد الحياة. وفي هذه البيئة الكئية والحزينة، عاش ماتيرلينك حياة غريبة وكان لايه خوف شديد من هذه الحياة الغريبة المخيفة ومن المجهول الذي يحمل في طياته رائحة الموت الذي يهد الإنسان ويقربه من مصيره المحتوم. فالموت له أوجه كثيرة غير مرئية، ولكنها محسوسة ومنتشرة في كل مكان من حولنا. ولا يستطيع الإنسان رؤية هذه الحقيقة بعينيه، ولكن يحسها بروحه. فللموت قوة خفية مرتبطة من وجهة نظر ماتيرلينك بالمكان. فالفضاء هو العنصر الأول الذي يرمز إلي العزلة والصمت والخوف في المسرح الأول لماتيرلينك وفيه يمنل المجهول كائناً مادياً مخيفاً، وغالباً ما يأخذ هذا المجهول صورة الموت الذي سيطر علي أعماله. فاستخدم بعض الآليات والأماكن التي تتير إلي الموت منتل الديكور، القصر ، البرج، الغابة، المحيط .. إلخ. ويهنم هذا العمل بدراسة الفضاء والإشارات إلي المجهول أو الموت في المسرح الأول لماتيرلينك. 